\title{
Pupil Size Measurement and Sucrose Ingestion for Quantifying and Decreasing Burden of Women during Mammography
}

\author{
Yongbum Lee, Mieko Uchiyama, Tomoko Sumiyoshi \\ Graduate School of Health Sciences, Niigata University, Niigata, Japan \\ Email: lee@clg.niigata-u.ac.jp
}

Received 17 September 2015; accepted 16 October 2015; published 19 October 2015

Copyright (C) 2015 by authors and Scientific Research Publishing Inc.

This work is licensed under the Creative Commons Attribution International License (CC BY). http://creativecommons.org/licenses/by/4.0/

(c) (i) Open Access

\section{Abstract}

Many women feel a physical burden, which usually appears as a pain, during mammography. We examined the pain-related pupil dilation response during mammography. Twenty-four healthy women participated in the examination. They were randomly assigned to water group or sucrose group. Water group and sucrose group ingested distilled water and a sucrose solution during mammography, respectively. Their pupil sizes were measured during mammography excluding $\mathrm{X}$-ray irradiation. The subjective intensity of pain during mammography was also assessed using visual analogue scales (VAS). The mean pupil diameter was $2.99 \mathrm{~mm}$ in relaxation phase before mammography, while it was $3.15 \mathrm{~mm}$ in a few seconds after breast compression (pressure phase). The difference between the two was statistically significant $(p<0.01)$. There was no correlation between the VAS scores and the pupil sizes. In the pressure phase, the mean pupil sizes of sucrose group and water group were $2.91 \mathrm{~mm}$ and $3.42 \mathrm{~mm}$, respectively. The difference between the two reached statistical significance $(p<0.01)$. These results suggest that we may be able to assess objectively the degree of pain associated with mammography and sweet taste stimuli may be effective to alleviate a women's burden received during mammography.

\section{Keywords}

Mammography, Pain Assessment, Pain Alleviation, Pupil Dilation, Sweet Stimuli

\section{Introduction}

Mammography is the most effective method available for the early diagnosis of breast cancer [1] and regular screening can significantly reduce mortality [2]. However, it is unfortunate that many women experience a pain 
during mammography [3]-[9] because the breast is compressed by parallel plates to make the breast tissue as even and thin as possible. In addition to the breast compression, the pain is due to the positioning required, such as twisting the neck and raising the arm. Appropriate techniques for radiography and management of the mammography device to obtain accurate images have been established. This has made it possible to obtain good radiographic images of the breast [10]-[13]. However, studies are lacking on objective methods to measure the physical burden on women and the alleviation of pain during mammography.

Several subjective assessment scales such as visual analogue scale (VAS) [14] [15] and McGill pain questionnaire (MPQ) [16] were used to predict the pain during mammography [6] [7]. The MPQ could be considered as the most famous pain assessment tool, but the process is a little complicated to obtain reliable MPQ scores. The VAS is perhaps the most widely used instrument for the measurement of pain intensity because it is a simple, robust, sensitive, and reproducible instrument. Furthermore, whereas the VAS takes only a few minutes, the MPQ requires about 15 minutes. Recently, muscle activities [17] [18] and sympathetic nervous activities [19] have been measured to estimate physical burden and psychological stress associated with mammography, respectively. These were objective assessment studies for quantification of pain or stress during mammography. The valuable objective assessment tools for quantifying the pain intensity should be further investigated. More research is therefore required using a validated pain measurement to establish the extent of pain and to establish the effect of this on future compliance [9].

As to the alleviation of pain during mammography, a few investigators have reported on the usefulness of radiolucent cushions [20] [21]. However, their use is debatable because of the high cost since these cushions are single-use. Patient-controlled compression was tested as a method to alleviate pain [22]. With this method, one of the breasts was compressed by a radiographer and the other was compressed by the participant. In $71 \%$ of the participants, self-compression resulted in significantly less pain than compression by a radiographer without a difference in the adequacy of the image quality and compression of the breast. It has been also reported that humorous stimuli would relieve the pain during mammography [23]. According to the report, the pain experienced during conventional mammography without a funny video was significantly greater than when they experienced during the same mammography but with the funny video. There are limited options currently available for alleviating pain related to mammography and further research will be needed to address this problem.

In the meantime, it has been reported that the pupil dilates markedly in response to increasing noxious stimulation in humans [24]. The magnitude of the pupil dilation response varies directly with intensity of noxious electrical fingertip stimulation [25]. These investigations suggest that the extent of pupil dilation response may provide a useful objective indicator for estimating pain quantitatively. Furthermore, it has been also reported that sucrose stimuli might induce the antinociceptive effects on pain in human [26] [27]. This suggestion indicates that an intake of sweet tastes during mammography might alleviate the pain related with mammography. We therefore examined the pain-related pupil dilation response during mammography with or without a sweet taste stimulus. The VAS was also used to assess subjective intensity of physical burden during mammography for this study. This study has two purposes. The first aim is to investigate whether the pupil-size change reflects the burden during mammography and the second aim is to test the usefulness of sweet taste stimuli for alleviation of the burden during mammography.

\section{Materials and Methods}

Volunteers were solicited for this study through poster advertisements. Twenty-four healthy women contacted us. Their mean age \pm standard deviation (SD) was $23.6 \pm 6.4$ years, height was $159.5 \pm 6.6 \mathrm{~cm}$, weight was 52.2 $\pm 5.6 \mathrm{~kg}$. Two of the 24 women had experienced mammography, and the remaining 22 had not. The participants were given explanation on the study objectives, methods, and safety, and their informed consent was obtained. This study was approved by the Research Ethics Committee of the Niigata University School of Medicine.

Observation in the study took place beginning at the end of positioning of the right breast, including compression, for mediolateral oblique (MLO) imaging but excluding X-ray irradiation. During the sham mammography, the Eye Mark Recorder (EMR-9; nac Image Technology Inc., Tokyo, Japan) was used to measure pupil sizes of subjects. The EMR is an eye tracking measurement and analysis system that has devices for measuring a subject's point of visual focus and pupil diameter. The device of EMR consists of a cap-type head unit and a control unit as shown in Figure 1. A pupil-corneal reflection method was used for eye detection in the system. The sampling rate was $60 \mathrm{~Hz}$. The detection resolution of pupil diameter was $0.01 \mathrm{~mm}$. Figure 2 shows a screenshot obtained from video output of the EMR. 

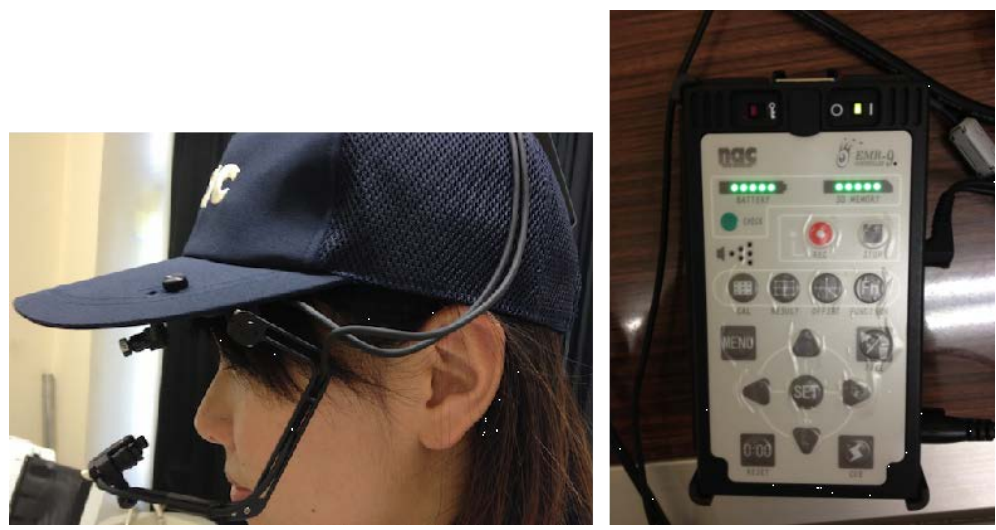

Figure 1. The Eye Mark Recorder used to measure pupil sizes (left: cap-type head unit, right: control unit).

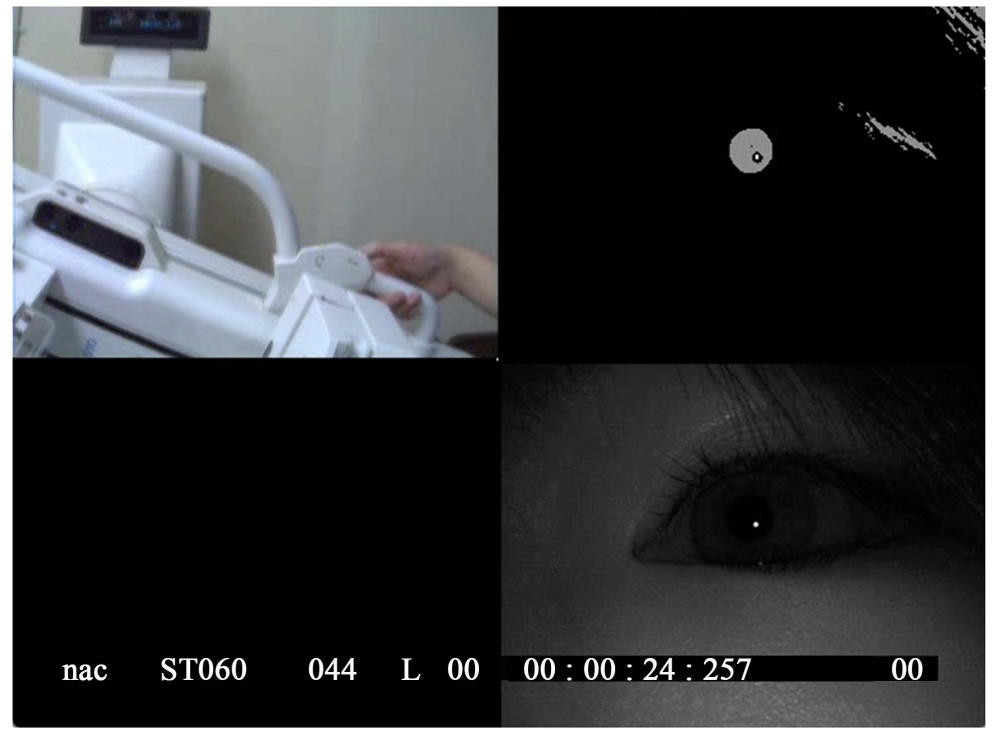

Figure 2. Screenshot captured from video output of the Eye Mark Recorder (upper left: view image, bottom right: eye image, upper right: binary image with pupil region automatically detected from eye image, bottom left: blank).

The pupil size of left eye was measured through the following four phases during mammography. The phase before positioning was defined as the Relaxation Phase (RP). The interval between the start of positioning and breast fixing was defined as the Keep Phase (KP). The interval between breast fixing and the end of imaging, which was actually sham because the participants were not irradiated by X-ray, was defined as the Pressure Phase (PP). The PP was further divided into two sections for data analysis. The first half and later half were defined as $\mathrm{PP}_{\mathrm{F}}$ and $\mathrm{PP}_{\mathrm{L}}$, respectively. The phase after the examination was defined as the After Phase (AP). For data analysis, we used pupil-size values obtained within some seconds from start of each phase. The numbers of seconds were approximately determined as one half of average time for each phase. Actually, they were 24,11 , 6 and 4 seconds for RP, KP, PP and AP, respectively.

The experimental procedure is shown in Figure 3. Subjects were randomly assigned to one of two treatment groups that differed according to whether they were to consume a sucrose solution (sucrose group, $\mathrm{n}=12$ ) or distilled water (water group, $\mathrm{n}=12$ ). Each of the subjects put a test paper impregnated with $3 \mathrm{ml}$ of a $24 \%$ weight/volume sucrose solution or of distilled water in her mouth, and kept it during mammography. The size of the test paper was $10 \times 1 \mathrm{~cm}$.

The subjective intensity of pain during mammography was also assessed using VAS. The VAS consisted of a horizontal line of $100 \mathrm{~mm}$ with scoring in increments of $1 \mathrm{~mm}$ from "no pain at all" at the leftmost position 


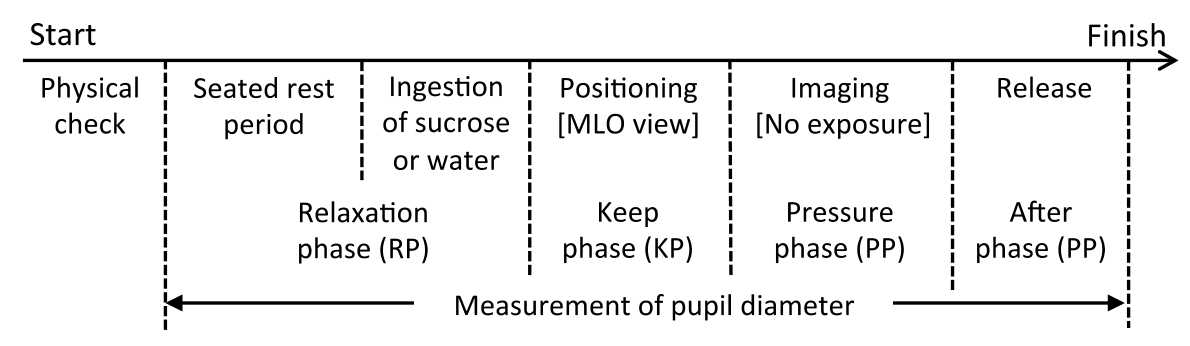

Figure 3. Experimental procedure.

(0 points) to "the worst pain you have ever felt" on the rightmost position (100 points). The participants were asked to point with their finger to the level of pain they were feeling during whole mammography.

\section{Results}

Figure 4 shows sequential binary images that represent pupil regions of a subject from RP to AP. The pupil region of $\mathrm{PP}_{\mathrm{F}}$ looks larger than that of other phases. Figure 5 shows a graph of mean pupil diameter obtained from all participants. Means and standard deviations (SDs) of pupil diameters in each phase are shown in Table 1. The mean pupil size of $\mathrm{PP}_{\mathrm{F}}$ for all subjects was obviously larger than the other phase's. The differences in the pupil diameters between $\mathrm{PP}_{\mathrm{F}}$ and other phases were statistically significant $(p<0.01)$. A significant difference was not recognized between sucrose group and water group in the entire phase during mammography but the pupil size of water group in $\mathrm{PP}_{\mathrm{F}}$ was significantly larger than sucrose group's $(p<0.01)$. Figure 6 is a plot diagram for VAS scores versus mean pupil diameters in $\mathrm{PP}_{\mathrm{F}}$. The correlation coefficient between the VAS scores and the pupil diameters was 0.19 . There was no significant difference between the two groups with respect to the VAS scores.

\section{Discussion}

Compressing breast usually gives strongest intensity of pain to woman undergoing mammography. Pupil dilation at $\mathrm{PP}_{\mathrm{F}}$ must be related with this matter. We supposed that we found out the pain-related pupil dilation response during mammography. This finding suggests that we may be able to assess objectively the degree of pain associated with mammography. However, the period that pupil size was dilating was short because there was no significant difference between $\mathrm{PP}_{\mathrm{L}}$ and other phases except $\mathrm{PP}_{\mathrm{F}}$ with respect to the pupil size. Instantaneous pain stimuli by breast compression would prompt a brief dilation of pupil. The relation between the intensity of pain and the degree of pupil dilation should be further investigated for acquisition of an objective assessment method for pain. The pupil change should be also compared to other objective indicators [17]-[19] for assessing the burden during mammography.

Although we were expecting a strong positive correlation between the VAS scores and the pupil diameters, there was no linear correlation or a weak linear correlation between the two. However, the pupil was evidently dilated by intense pain associated with breast compression during mammography. The experimental data suggest that women undergoing mammography may have been receiving an unconscious physical burden even if they do not recognize the burden as pain. $\mathrm{In}_{\mathrm{PP}}$, the mean pupil size of sucrose group was smaller than water group's. That is to say, the pupil dilation response in $\mathrm{PP}_{\mathrm{F}}$ was significantly suppressed by ingestion of a sucrose solution. These results suggest that sweet taste stimuli using sucrose are effective to relieve the unconscious physical burden received during mammography. It would be necessary to further investigate for acquisition of information about more effective intensity of sweet taste stimuli. We know that the subjective and objective data do not always match but one reason of the inconsistency may be due to the small number of participants. Therefore, the additional recruitment of subjects would be also necessary to our future works.

\section{Conclusion}

Pupil sizes of 24 women were measured to investigate pain-related pupil dilation response during mammography. The pupil sizes in a few seconds after breast compression were obviously larger than those of relaxation phase before mammography. This result may give us to quantitatively estimate the degree of pain associated 


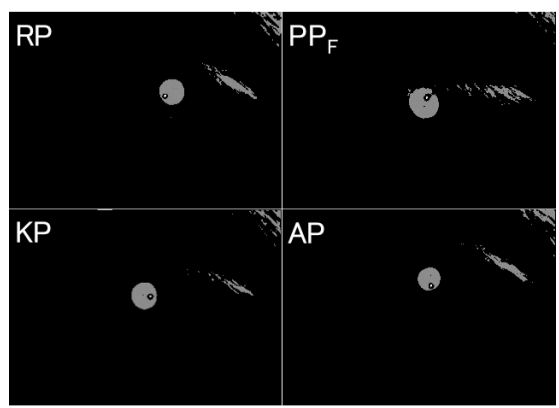

Figure 4. Sequential binary images represented pupil regions of a subject.

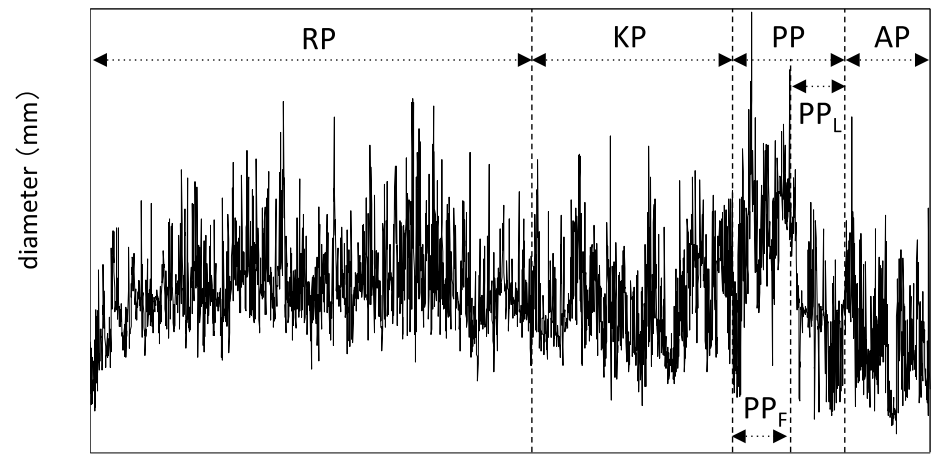

Figure 5. Fluctuating mean pupil diameter obtained from all subjects.

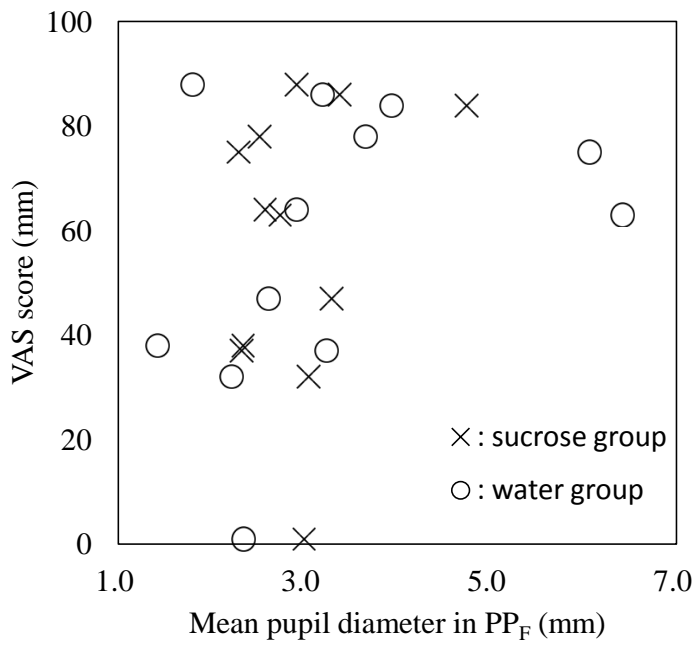

Figure 6. Plot diagram for VAS scores versus pupil diameters in $\mathrm{PP}_{\mathrm{F}}$.

Table 1. Pupil diameters (mm) measured during mammography.

\begin{tabular}{|c|c|c|c|c|c|c|}
\hline & $\mathrm{RP}$ & KP & $\mathrm{PP}_{\mathrm{F}}$ & $\mathrm{PP}_{\mathrm{L}}$ & $\mathrm{AP}$ & Entire phase \\
\hline All subjects & $2.99 \pm 0.13$ & $2.94 \pm 0.14$ & $3.15 \pm 0.19$ & $2.92 \pm 0.15$ & $2.81 \pm 0.15$ & $2.98 \pm 0.12$ \\
\hline Sucrose group & $3.08 \pm 0.20$ & $2.94 \pm 0.16$ & $2.91 \pm 0.19$ & $2.81 \pm 0.07$ & $2.72 \pm 0.18$ & $2.98 \pm 0.22$ \\
\hline Water group & $2.91 \pm 0.16$ & $2.95 \pm 0.25$ & $3.42 \pm 0.34$ & $3.05 \pm 0.31$ & $2.90 \pm 0.25$ & $2.97 \pm 0.26$ \\
\hline
\end{tabular}

Values are presented as mean \pm standard deviation (SD). 
with mammography in the near future. The subjects held a sucrose solution or distilled water in their mouth during mammography. The pupil dilation response was significantly suppressed by the ingestion of the sucrose solution. Sweet taste stimuli may be effective to alleviate a physical burden received during mammography. Visual analogue scale (VAS) was also used to assess subjective intensity of pain during mammography. There was no correlation between the VAS scores and the pupil sizes. Further investigations should be conducted for quantitative assessment and alleviation of pain during mammography.

\section{Acknowledgements}

The authors thank Ms. Kanae Nagai for assisting with experiments and data analysis. This study was supported by JSPS KAKENHI Grant Number 26463382.

\section{References}

[1] Drukker, B.H. (1997) Breast Disease: A Primer on Diagnosis and Management. International Journal of Fertility and Women's Medicine, 42, 278-287.

[2] Kerlikowske, K., Grady, D., Rubin, S.M., Sandrock, C. and Ernster, V.L. (1995) Efficacy of Screening Mammography. A Meta-Analysis. Journal of the American Medical Association, 273, 149-154. http://dx.doi.org/10.1001/jama.1995.03520260071035

[3] Jackson, V.P., Lex, A.M. and Smith, D.J. (1988) Patient Discomfort during Screen-Film Mammography. Radiology, 168, 421-423. http://dx.doi.org/10.1148/radiology.168.2.3393659

[4] Aro, A.R., Absetz-Ylöstalo, P., Eerola, T., Pamilo, M. and Lönnqvist, J. (1996) Pain and Discomfort during Mammography. European Journal of Cancer, 32, 1674-1679. http://dx.doi.org/10.1016/0959-8049(96)00140-2

[5] Keemers-Gels, M.E., Groenendijk, R.P., Van Den Heuvel, J.H., Boetes, C., Peer, P.G. and Wobbes, T.H. (2000) Pain Experienced by Women Attending Breast Cancer Screening. Breast Cancer Research and Treatment, 60, $235-240$. http://dx.doi.org/10.1023/A:1006457520996

[6] Hafslund, B. (2000) Mammography and the Experience of Pain and Anxiety. Radiography, 6, 269-272. http://dx.doi.org/10.1053/radi.2000.0281

[7] Sharp, P.C., Michielutte, R., Freimanis, R., Cunningham, L., Spangler, J. and Burnette, V. (2003) Reported Pain Following Mammography Screening. Archives of Internal Medicine, 163, 833-836. http://dx.doi.org/10.1001/archinte.163.7.833

[8] Asghari, A. and Nicholas, M.K. (2004) Pain during Mammography: The Role of Coping Strategies. Pain, 108, 170179. http://dx.doi.org/10.1016/j.pain.2003.12.022

[9] Davey, B. (2007) Pain during Mammography: Possible Risk Factors and Ways to Alleviate Pain. Radiography, 13, 229-234. http://dx.doi.org/10.1016/j.radi.2006.03.001

[10] Haus, A.G. (2002) Historical Technical Developments in Mammography. Technology in Cancer Research and Treatment, 1, 119-126. http://dx.doi.org/10.1177/153303460200100204

[11] Ng, K.H., Jamal, N. and DeWerd, L. (2006) Global Quality Control Perspective for the Physical and Technical Aspects of Screen-Film Mammography_-Image Quality and Radiation Dose. Radiation Protection Dosimetry, 121, 445-451. http://dx.doi.org/10.1093/rpd/ncl051

[12] Robson, K.J. (2010) Advances in Mammographic Imaging. British Journal of Radiology, 83, 273-275. http://dx.doi.org/10.1259/bjr/97865299

[13] Yamada, T. (2010) Current Status and Issues of Screening Digital Mammography in Japan. Breast Cancer, 17, 163168. http://dx.doi.org/10.1007/s12282-009-0191-z

[14] Keel, K.D. (1948) The Pain Chart. The Lancet, 2, 6-8. http://dx.doi.org/10.1016/S0140-6736(48)91787-5

[15] Turk, D.C. and Melzack. R. (2001) Handbook of Pain Assessment. 2nd Edition, The Guilford Press, New York.

[16] Merzack, R. (1975) The McGill Pain Questionnaire: Major Properties and Scoring Methods. Pain, 1, 277-299. http://dx.doi.org/10.1016/0304-3959(75)90044-5

[17] Uchiyama, M., Lee, Y., Sadakata, M., Sayama, M. and Tsai, D.Y. (2012) Measurement of Muscle Activities for Evaluating Physical Burden and Pain during Mammography Positioning. Tohoku Journal of Experimental Medicine, 228, 53-58. http://dx.doi.org/10.1620/tjem.228.53

[18] Uchiyama, M., Lee, Y., Sadakata, M., Tsai, D.Y. and Sayama, M. (2015) Quantification of the Pain and Physical Burden Experienced during Positioning for Craniocaudal Imaging in Mammography, Evaluated by Measurement of Muscle Activity. Health, 7, 23-34. http://dx.doi.org/10.4236/health.2015.71004 
[19] Uchiyama, M., Lee, Y., Sadakata, M., Kazama, K., Minagawa, Y. and Tsurumaki, M. (2013) Effects of Mammography Positioning on the Autonomic Nervous Function. Health, 5, 1335-1341. http://dx.doi.org/10.4236/health.2013.58181

[20] Tabar, L., Lebovic, G.S., Hermann, G.D., Kaufman, C.S., Alexander, C. and Sayre, J. (2004) Clinical Assessment of a Radiolucent Cushion for Mammography. Acta Radiologica, 45, 154-158. http://dx.doi.org/10.1080/02841850410003978

[21] Markle, L., Roux, S. and Sayre, J.W. (2004) Reduction of Discomfort during Mammography Utilizing a Radiolucent Cushioning Pad. Breast Journal, 10, 345-349. http://dx.doi.org/10.1111/j.1075-122X.2004.21352.X

[22] Kornguth, P.J., Rimer, B.K., Conaway, M.R., Sullivan, D.C., Catoe, K.E., Stout, A.L. and Brackett, J.S. (1993) Impact of Patient-Controlled Compression on the Mammography Experience. Radiology, 186, 99-102. http://dx.doi.org/10.1148/radiology.186.1.8416595

[23] Lee, Y. and Uchiyama, M. (2015) The Effect of Humorous Stimuli on Alleviating Pain during Mammography: A Preliminary Study. Health, 7, 659-664. http://dx.doi.org/10.4236/health.2015.76078

[24] Oka, S., Chapman, C.R., Kim, B., Nakajima, I., Shimizu, O. and Oi, Y. (2007) Pupil Dilation Response to Noxious Stimulation: Effect of Varying Nitrous Oxide Concentration. Clinical Neurophysiology, 118, 2016-2024. http://dx.doi.org/10.1016/j.clinph.2007.04.023

[25] Chapman, C.R., Oka, S., Bradshaw, D.H., Jacobson, R.C. and Donaldson, G.W. (1999) Phasic Pupil Dilation Response to Noxious Stimulation in Normal Volunteers: Relationship to Brain Evoked Potentials and Pain Report. Psychophysiology, 36, 44-52. http://dx.doi.org/10.1017/S0048577299970373

[26] Bhattacharjee, M. and Mathur R. (2005) Antinociceptive Effect of Sucrose Ingestion in the Human. Indian Journal of Physiology Pharmacology, 49, 383-394.

[27] Kakeda, T. (2010) Potential of Sucrose-Induced Analgesia to Relieve Pain in Male Adults: A Preliminary Study. Japan Journal of Nursing Science, 7, 169-173. 